\title{
SHORT REPORT: DETECTION OF 72-75-kD and 123-kD FRACTIONS OF LEISHMANIA ANTIGEN IN URINE OF PATIENTS WITH VISCERAL LEISHMANIASIS
}

\author{
M. DE COLMENARES, M. PORTUS, C. RIERA, M. GAllego, M. J. AISA,
} S. TORRAS, AND C. MUNOZ

\begin{abstract}
Laboratori de Parasitologia, Departament de Microbiologia i Parasitologia Sanitaries, Facultat de Farmacia, Universitat de Barcelona, Barcelona, Spain; Servei de Microbiologia, Hospital de la Santa Creu i Sant Pau, Barcelona, Spain
\end{abstract}

\begin{abstract}
Two polypeptide fractions of $72-75 \mathrm{kD}$ were detected in the urine of 14 of 15 patients with visceral leishmaniasis (VL) and another fraction of $123 \mathrm{kD}$ was found in 10 of the 15 patients by using a Western blot technique. None of these fractions was detected in the urine of 20 controls. These results suggest that antigen detection in urine could be a powerful, noninvasive method for VL diagnosis.
\end{abstract}

An increasing number of cases of leishmaniasis in patients infected with the human immunodeficiency virus (HIV) and the low humoral response found in these individuals urges the development of new diagnostic methods based on antigen detection. Noninvasive techniques may be of great value in epidemiologic studies and antigen detection in urine is a good option if the technique is sufficiently sensitive and specific.

Detection of Leishmania antigen in the urine of patients with visceral leishmaniasis (VL) was performed by Kohanteb and others in $1987^{\prime}$ using agar precipitation techniques. In 1992, Dourado and others (unpublished data) presented preliminary results of Leishmania antigen detection in urine using an immunocapture technique. In neither case were the detected antigens identified.

The work reported here was undertaken to develop a method to detect Leishmania antigen in urine for use as a diagnostic tool. Preliminary unpublished experiments using a dot-enzyme-linked immunosorbent assay (ELISA) failed to give satisfactory results; a small proportion of healthy controls and some patients with acquired immunodeficiency syndrome (AIDS), but no history of leishmaniasis, gave positive results. For this reason, we assessed the utility of a Western blot technique in the identification of the specific polypeptide fractions in urine.

Urine samples from 35 individuals were studied, including 15 from clinically proven cases of VL (nine HIV-positive and six HIV-negative) and 20 from negative control patients (eight HIV-positive and $12 \mathrm{HIV}$-negative) who had no history of leishmaniasis. Visceral leishmaniasis was diagnosed on the basis of clinical symptoms, including intermittent fever, hepatosplenomegaly, pancytopenia, and anemia, and on the detection of Leishmania amastigotes in bone marrow aspirates. Antibodies in serum were detected using a dot-ELISA and an ELISA in all HIV-negative patients and in seven of nine HIV-positive patients with VL. Urine samples were collected and stored in aliquots at $-40^{\circ} \mathrm{C}$ until use; $15 \mathrm{ml}$ of the urine sample was concentrated using Centripep-10 Concentrators (\#4304; Amicon, Beverly, MA) through three consecutive centrifugations of 40,10 , and $5 \mathrm{~min}$ at $2,800 \times$ $g$ at room temperature. The final volume obtained was 0.6 $1 \mathrm{ml}$, depending on the amount of sediment.
Leishmania hyperimmune serum (LHS) was obtained by immunization of rabbits with two subcutaneous inoculations of $2.5 \times 10^{8}$ promastigotes (MHOM/FR/78/LEM 75) emulsified in Freund's incomplete adjuvant (Sigma, St. Louis, MO). These were given one month apart. The rabbits were given booster injections seven, 14 , and 21 days after the final immunization with an intravenous inoculation of $1 \times 10^{8}$, $1.6 \times 10^{8}$, and $2 \times 10^{8}$ promastigotes in phosphate-buffered saline. Immunization was monitored by dot-ELISA and rabbits were bled one week after the final inoculation. Serum was separated and the immunoglobulin fraction was obtained by ammonium sulfate precipitation.

Rabbit IgG antisera (R-3382; Sigma) was biotinylated using N-hydroxysuccinimidobiotin (H-1759; Sigma). Proteins were separated on a $7.5 \%$ gel by sodium dodecyl sulfatepolyacrylamide gel electrophoresis (SDS-PAGE) under reducing conditions. A Leishmania promastigote suspension (LEM 75) was used as a positive control. Molecular weights were estimated from the relative mobility of simultaneously run standard proteins of known molecular mass (SDS-PAGE Molecular Weight Standards, low range; Bio-Rad, Richmond, CA).

Polypeptides were transblotted onto nitrocellulose sheets (0.45- $\mu \mathrm{m}$ pore size, HAWP 304 FO; Millipore, Bedford, MA) and the sheets were blocked with $20 \mathrm{mM}$ Tris, 0.13 $\mathrm{mM} \mathrm{NaCl}, \mathrm{pH} \mathrm{7.4,} \mathrm{(TS)-5 \%} \mathrm{skim} \mathrm{milk} \mathrm{for} 30 \mathrm{~min}$. All incubations were carried out at room temperature (approximately $25^{\circ} \mathrm{C}$ ). The nitrocellulose sheets were then incubated with a 1:500 dilution of LHS in TS, $0.05 \%$ Tween 20 (TST)$1 \%$ skim milk (TST-M) for $3 \mathrm{hr}$. The sheets were then washed and incubated with antirabbit biotinylated serum diluted 1:200 in TST-M for $1 \mathrm{hr}$, then washed and incubated with $2.5 \mu \mathrm{g} / \mathrm{ml}$ of avidin-peroxidase (A-3151; Sigma) in TST for $30 \mathrm{~min}$. Color was developed with (3,3'-diaminobenzidine tetrahydrochloride, D-5637; Sigma) $/ \mathrm{H}_{2} \mathrm{O}_{2}$ and reaction was stopped with tap water after $30 \mathrm{~min}$.

Immunoblot analysis showed that LHS recognized two polypeptide fractions of $72-75 \mathrm{kD}$ in the urine of 14 of 15 patients with VL and another component of approximately $123 \mathrm{kD}$ in 10 of these cases; the fractions were not identified in the urine of either normal controls or VL-negative AIDS patients (Figure 1 and Table 1). Nevertheless, LHS recog- 


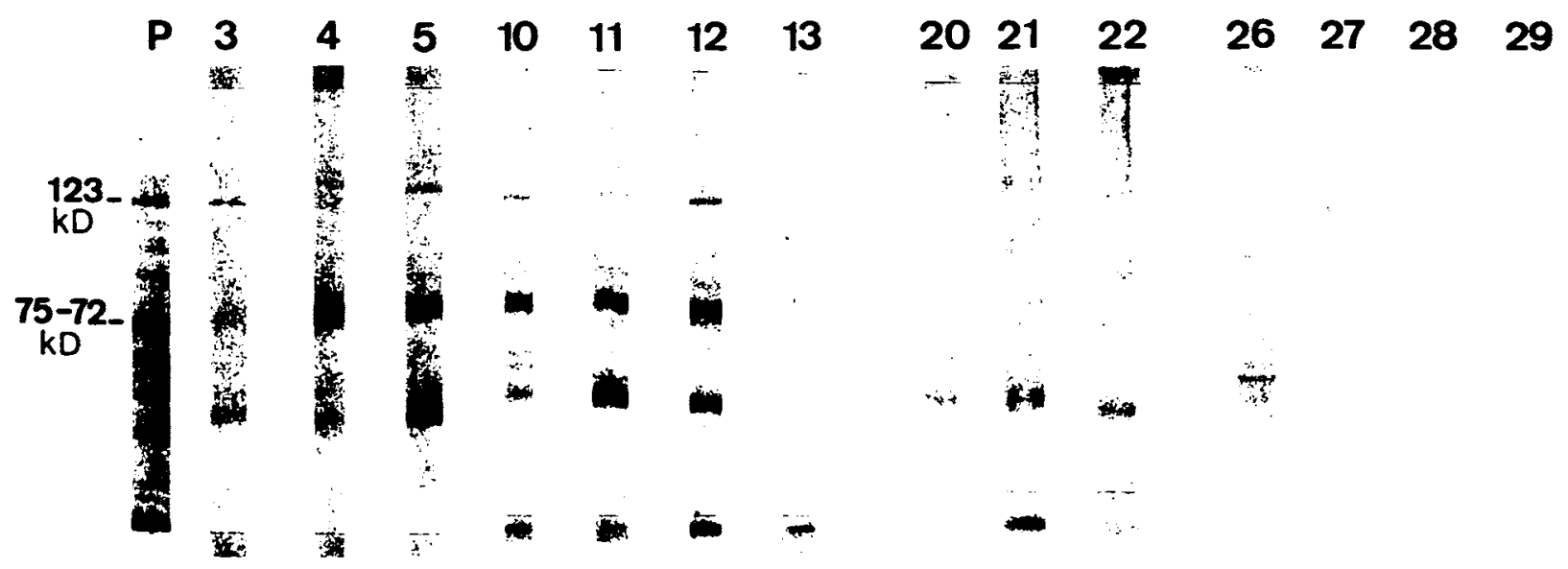

FigURE 1. Immunoblot analysis of the urine of patients with visceral leishmaniasis (lanes 3-5, human immunodeficiency virus (HIV)positive; lanes 10-13 HIV-negative) and negative controls (lanes 20-22, HIV-positive; lanes 26-29, HIV-negative). Promastigotes (lane P) were used as a positive control. $\mathrm{kD}=$ kilodaltons.

nized a 50-kD fraction and other fractions with high molecular weights $(>150 \mathrm{kD})$ in almost all urine samples, which may responsible for the false-positive results detected in preliminary studies with a dot-ELISA.

In four of the five patients in which the 123-kD component was not detected (no. 6, 7, 13, and 14), the samples were collected during treatment with Glucantime ${ }^{\circledR}(\mathrm{N}$-metilglucamine antimoniate; Rhone-Poulenc Rorer S.A., Alcorcon, Madrid, Spain). Furthermore, the one individual (a child, no. 15) in whom the double band of 72-75 kD was not detected was also currently undergoing treatment for leishmaniasis. Consequently, the lack of antigen may coincide with observations of Kohanteb and others, ' who did not find antigen in the urine of two kala-azar patients after 10 and 13 days of treatment with Glucantime ${ }^{\text {. Jaffe and others }}{ }^{2}$ described two monoclonal antibodies that recognize a double band of $69-73 \mathrm{kD}$ in the membrane of the different species

TABLE 1

Polypeptide fractions in the urine of patients with visceral leishmaniasis

\begin{tabular}{|c|c|c|c|}
\hline \multirow{2}{*}{$\begin{array}{c}\text { Patient } \\
\text { no. }\end{array}$} & \multirow{2}{*}{$\begin{array}{c}\text { HIV } \\
\text { status* }\end{array}$} & \multicolumn{2}{|c|}{ Antigens detected in urinet } \\
\hline & & $123 \mathrm{kD}$ & $72-75 \mathrm{kD}$ \\
\hline 1 & + & ++ & ++ \\
\hline 2 & + & + & + \\
\hline 3 & + & $++t$ & ++ \\
\hline 4 & + & + & $++t$ \\
\hline 5 & + & +++ & $++t$ \\
\hline 6 & + & - & ++ \\
\hline 7 & + & - & + \\
\hline 8 & + & - & + \\
\hline 9 & + & ++ & + \\
\hline 10 & - & $++t$ & +++ \\
\hline 11 & - & $++t$ & $++t$ \\
\hline 12 & - & +++ & $++t$ \\
\hline 13 & - & - & $+t$ \\
\hline 14 & - & - & + \\
\hline 15 & - & + & - \\
\hline
\end{tabular}

* HIV = human immunodeficiency virus.

$+-=$ not detectable: $+=$ weak signat: $++=$ strong signal: $+++=$ very strong signal. of the $L$ donovani complex, but not in other species of the genus. ${ }^{3}$

The results of this preliminary study suggest that the detection of polypeptide fractions of $72-75 \mathrm{kD}$ and $123 \mathrm{kDa}$ of the Leishmania antigen in urine may be of value in the development of a direct antigen diagnostic test.

Acknowledgments: This study was possible thanks to the generous collaboration of the Services of Internal Medicine and Microbiology of the following hospitals: Hospital Clinic i Provincial (Barcelona), Hospital Germans Trias i Pujol (Badalona), Hospital de Sant Camil (Sant Pere de Ribes), Hospital de Sant Joan de Deu (Barcelona), Hospital de la Santa Creu i Sant Pau (Barcelona), Residencia Sanitaria de Bellvitge (Hospitalet de Llobregat), and Residencia Sanitaria de la Vall d'Hebron (Barcelona). We also thank Dr. D. McMahon-Pratt for reading the manuscript and suggestions.

Financial support: This research was supported by Plan Nacional de I + D. Project CICYT SAL 90-0960-CO2-01.

Authors' addresses: M. de Colmenares, M. Portus, C. Riera, M. Gallego, M. J. Aisa, and S. Torras, Laboratori de Parasitologia, Departament de Microbiologia i Parasitologia Sanitaries, Facultat de Farmacia, Universitat de Barcelona, Avda. Diagonal s/n, 08028 Barcelona, Spain. C. Munoz, Servei de Microbiologia, Hospital de la Santa Creu i Sant Pau, Avda. Sant Antoni Maria Claret 167, 08025 Barcelona, Spain.

Reprint requests: M. de Colmenares, Laboratori de Parasitologia, Facultat de Farmacia, Avda. Diagonal s/n, 08028 Barcelona, Spain.

\section{REFERENCES}

1. Kohanteb J, Ardehali SM, Rezai HK, 1987. Detection of Leishmania donovani antigen and antibody in the urine of visceral leishmaniasis patients. Trans $R$ Soc Trop Med Hyg 81: 578580.

2. Jaffe CL, Bennett E, Grimaldi G Jr, McMahon-Pratt D, 1984. Production and characterization of species-specific monoclonal antibodies against Leishmania donovani for immunodiagnosis. I Immunol 133: 440-447.

3. Jaffe CL, McMahon-Pratt D, 1987. Serodiagnostic assay for visceral leishmaniasis employing monoclonal antibodies. Trans $R$ Soc Trop Med Hyg 81: 587-594. 